\title{
Large-scale neural dynamics: Simple and complex
}

\author{
S Coombes*
}

December 23, 2009

\begin{abstract}
We review the use of neural field models for modelling the brain at the large scales necessary for interpreting EEG, fMRI, MEG and optical imaging data. Albeit a framework that is limited to coarse-grained or mean-field activity, neural field models provide a framework for unifying data from different imaging modalities. Starting with a description of neural mass models we build to spatially extended cortical models of layered two-dimensional sheets with long range axonal connections mediating synaptic interactions. Reformulations of the fundamental non-local mathematical model in terms of more familiar local differential (brain wave) equations are described. Techniques for the analysis of such models, including how to determine the onset of spatio-temporal pattern forming instabilities, are reviewed. Extensions of the basic formalism to treat refractoriness, adaptive feedback and inhomogeneous connectivity are described along with open challenges for the development of multi-scale models that can integrate macroscopic models at large spatial scales with models at the microscopic scale.
\end{abstract}

\section{Introduction}

Systems of equations that can model the brain at the very large scale are becoming increasingly important for underpinning experimental techniques including those of EEG, fMRI, MEG and optical imaging using voltage sensitive dyes. All of these can reveal patterns of spatio-temporal activity that span centimetres

\footnotetext{
*Department of Mathematical Sciences, University of Nottingham, Nottingham, NG7 2RD, UK. email: stephen. coombes@nottingham.ac.uk
}

of tissue in the cerebral cortex. One brute-force approach to building models that can help make sense of these rich dynamical patterns is to consider the detailed properties of synapses, neurons, microcircuits and cortical columnar organisation and build vast computational compartmental models [1]. Although such an approach may bring one close to the neurobiology, the complexity of such models is a severe barrier to gaining insight into emergent neurodynamics. An alternative approach is to consider coarse-grained cortical units, called neural masses, and use these as building blocks for cortical tissue models. Such an approach has two main advantages. Firstly, as opposed to the brute-force approach there is a drastic reduction in the dimensionality of both the parameter and variable space. Secondly, since detailed knowledge of single neuron properties (ionic currents and dendritic structure) is not required, much of the experimental data (on connectivity, neurotransmitter types, distribution of axonal speeds) needed to constrain such models is already available. The current mathematical approach for understanding coarse-grained activity of large ensembles of neurons in cortex is based around the work of Wilson and Cowan [2,3], Amari [4, 5] and Nunez [6] in the 1970s. Because the number of neurons and synapses in even a small piece of cortex is so vast a natural first approximation is to take a continuum limit and treat cortical space as continuous, giving rise to the notion of a neural field model. These models typically take the form of integro-differential equations. Their non-local nature (arising from long range axonal connections) has led to the development of a set of analytical and numerical tools for the study of waves and patterns based around natural extensions of those used for local partial differential equa- 
tion (PDE) models. Indeed they have been used in a number of neural contexts including understanding mechanisms for short term working memory [7], motion perception [8], representations in the headdirection system [9], and feature selectivity in the visual cortex [10]. For a review of such models in both one and two dimensions that can incorporate realistic forms of axo-dendritic interactions we refer the reader to $[11,12]$. Albeit a framework that is limited to coarse-grained or mean-field activity, neural field models provide a direct connection from neural activity to EEG and fMRI data [13, 14] (unifying data from different imaging modalities) as well as a providing a bridge to cognitive theories of brain function [15]. Moreover, dynamic causal modelling (DCM) (see [16] for a review), which is frequently invoked for the interpretation of $\mathrm{fMRI}$ data, is now being extended from a data-driven perspective to incorporate activity models based upon neural field equations [17]. In light of the recent and rapid advances in the imaging of large scale cortical dynamics it is thus timely to review how neural field models can underpin empirical research that emphasises brain structure, dynamics and function.

We begin this overview of the practical uses of neural field models by first describing their basic building block, namely a neural mass model of a homogeneous neuronal population (which we may loosely think of as being a part of a cortical column). Next we describe the dynamics of an interacting set of neural masses (now at the scale of a whole cortical column) and explore their dynamics using bifurcation theory to uncover the natural time-scales for emergent rhythms. Focusing on the Liley et al. model [18], we review the success of such descriptions in generating oscillations consistent with the alpha band of the human EEG spectrum. Extensions to this population model to treat refractoriness and spike-frequency adaptation are also discussed. Next we show to model a cortical area as a two-dimensional continuous network of such cortical columns, defining a neural field. After reviewing the basic instability mechanism that can lead to the formation of travelling patterns of activ- ity we show how to formulate the model in terms of a PDE, recovering the Jirsa-Haken-Nunez brain wave equation in one spatial dimension [19]. In two spatial dimensions we show how the full non-local dynamics of a neural field model can be approximated with a local PDE model and consider its extension to treat patchy connections of the type that arise when isotropic connectivity is periodically modulated. We also discuss the effects that more general inhomogeneous connectivities can have on wave propagation through cortex, using mathematical analysis to emphasise the conditions for wave propagation failure. As an exemplar of the gains to be made with coarsegrained modelling we report on recent work of Bojak et al. [13] that utilises neural field models with realistic anatomical and physiological parameters for a folded cortex and a realistic head model to predict EEG and fMRI responses. Finally we discuss future directions for the mathematical descriptions of neural tissue relevant to neuroimaging.

\section{Population modules}

It is common practice to define a neural mass as a collection of thousands of near identical interconnected neurons with a preference to operate in synchrony. The spatial extent of this population is taken to be on the order of a few hundred micrometers. The state variable describing the activity of the population is the average membrane potential. Perhaps the most well known neural mass model is that of Jansen and Rit [20] based on the original work of Lopes Da Silva et al. [21]. Such lumped parameter models are capable not only of producing EEG style alpha rhythms but can generate more complex signals ranging from delta to gamma seen in EEG and MEG recordings (by appropriately modifying the population kinetics within a physiologically plausible range) [22]. Moreover they are amenable to mathematical analysis using techniques from dynamical systems theory and notably bifurcation theory [23]. However, it is well to mention here that because of the "near to synchrony" assumption, neural mass models are unable to say 


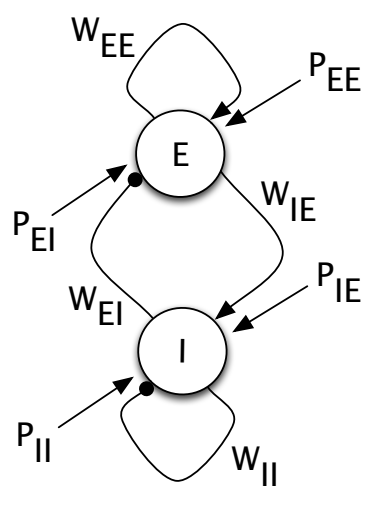

Figure 1: A diagram of a local cortical module represented by the interaction of two neuronal populations, one excitatory $(E)$ and the other inhibitory $(I)$.

anything about more complex behaviours within a single population, such as phase-locked states (away from synchrony) or clustering. Recent approaches that improve upon this situation have been developed by Stefanescu and Jirsa (using mode decomposition techniques) [24] and Laing and Kevrekidis (using "equation free modelling" and generalised polynomial chaos expansions) $[25,26]$.

One of the more successful population models for generating rhythms consistent with those found in the human EEG spectrum is that of Liley et al. [18]. In this mesoscopic model cortical activity is locally described by the mean soma membrane potentials of an interacting excitatory and an inhibitory population. The interaction is through a model of the synapse that treats both shunting currents and a realistic time course for post-synaptic conductance changes. Referring to the diagram in Fig. 1 the model can be written in a succinct form as

$$
\tau_{a} \dot{a}=-a+\sum_{b} W_{a b}\left(h_{b}-a\right),
$$

with $a, b \in\{E, I\}$, where $E(I)$ is the mean membrane potential in the excitatory (inhibitory) population. The relaxation time constants for the populations are given by $\tau_{a}$, whilst $h_{a}$ describes a reversal potential such that $h_{E}\left(h_{I}\right)$ is positive (negative) with respect to the resting state. The weights $W_{a b}$ are the product of a static strength factor and a dynamic con- ductance $W_{a b}=\bar{W}_{a b} g_{a b}$, where

$$
Q_{a b} g_{a b}=f_{b}\left(b\left(t-\Delta_{b a}\right)\right)+P_{a b} .
$$

Here $Q_{a b}$ represents a linear differential operator:

$$
Q_{a b}=\left(1+\frac{1}{\alpha_{a b}} \frac{\mathrm{d}}{\mathrm{d} t}\right)^{2},
$$

and the conductances are considered to be driven by a combination of firing from populations to which they are connected and some external drive. The former is modelled using a sigmoidal function:

$$
f_{a}(z)=\frac{1}{1+\mathrm{e}^{-\beta_{a}\left(z-\theta_{a}\right)}}, \quad \beta_{a}>0,
$$

and the latter, $P_{a b}$, is considered constant. Note the inclusion of delays $\Delta_{b a}$ in (2) that represent the fixed axonal communication lag for action potentials propagating from population $b$ to $a$. Exploiting the linearity of $Q_{a b}$ the model for the conductance can be integrated to give $g_{a b}=\eta_{a b} *\left[f_{b}+P_{a b}\right]$, where $*$ denotes a temporal convolution: $(\eta * f)(t)=\int_{0}^{t} \eta(t-s) f(s) \mathrm{d} s$ and

$$
\eta_{a b}\left(t+\Delta_{a b}\right)=\alpha_{a b}^{2} t \mathrm{e}^{-\alpha_{a b} t} H(t),
$$

where $H$ is a Heaviside step function. We recognise $\eta_{a b}(t)$ as a delayed $\alpha$-function, commonly used in computational neuroscience to mimic the rise and fall of a post-synaptic conductance change.

To determine the properties of such a population model it is natural to first consider the stability of the steady state, which we shall denote by $a_{\mathrm{ss}}$ (defined by $\dot{a}=0$ ). Linearising about this fixed point and seeking solutions of the form $\mathrm{e}^{\lambda t}$ gives a characteristic equation $\operatorname{det} \mathcal{E}(\lambda)=0$ where the entries of the $2 \times 2$ matrix $\mathcal{E}(\lambda)$ are

$$
[\mathcal{E}(\lambda)]_{a b}=\left(\lambda \tau_{a}+\kappa_{a}\right) \delta_{a b}-\widehat{W}_{a b} \widetilde{\eta}_{a b}(\lambda) .
$$

Here $\widehat{W}_{a b}=\bar{W}_{a b}\left(h_{b}-a_{\mathrm{ss}}\right) f_{b}^{\prime}\left(b_{\mathrm{ss}}\right), \delta_{a b}$ is the Kroneckerdelta, $\kappa_{a}=1+\sum_{b} \bar{W}_{a b} f_{b}\left(b_{\mathrm{ss}}\right)$ and $\widetilde{\eta}_{a b}(\lambda)$ is a Laplace transform given by:

$$
\int_{0}^{\infty} \mathrm{d} s \eta_{a b}(s) \mathrm{e}^{-\lambda s}=\frac{\mathrm{e}^{-\lambda \Delta_{b a}}}{\left(1+\lambda / \alpha_{a b}\right)^{2}} .
$$

It is instructive to consider the limit $\tau_{a} \rightarrow 0, \alpha_{a b}=\alpha$, $\kappa_{a}=\kappa$ and $\Delta_{a b}=\Delta$ in which case the characteristic equation takes the simple form

$$
\kappa(1+\lambda / \alpha)^{2}=\Gamma_{ \pm} \mathrm{e}^{-\lambda \Delta},
$$


where $\Gamma_{ \pm}$are the two eigenvalues of $\widehat{W}$. If a pair of complex conjugate eigenvalues $\lambda=\nu \pm i \omega$ crosses the imaginary axis $\nu=0$ from left to right in the complex plane, then a Hopf bifurcation can occur, leading to the formation of periodic oscillations. This is almost generic in the presence of delays, see for example [27]. For an interesting discussion on the role of delays in models for generalised epileptic seizures we refer the reader to Breakspear et al. [28]. For zero-delay $(\Delta=0)$ let us suppose that $\widehat{W}$ has a pair of complex conjugate eigenvalues $r \mathrm{e}^{ \pm i \theta}$ with $0<\theta<$ $\pi$. In this case a Hopf bifurcation occurs when $1=$ $\sqrt{r / \kappa} \cos (\theta / 2)$ independent of $\alpha$ with a non-zero frequency $\omega=\alpha \sqrt{r / \kappa} \sin (\theta / 2)$. However, in general a Hopf bifurcation will depend on the relative timescales in the model and should be determined as the solution of $\operatorname{det} \mathcal{E}(i \omega)=0$. In practice it is much easier to numerically determine the stability of fixed points of the full ten dimensional model, defined by equations (1-5), using software such as XPPAUT [29]. Moreover, this allows us to construct bifurcation diagrams like those in Fig. 2, which shows the coexistence of a large and small amplitude periodic orbit, with time-course shown in Fig. 3. The large amplitude, $(\sim 5$ $\mathrm{Hz}$ ) orbit has been suggested to correspond to a form of epileptic dynamics, whilst the smaller amplitude $(\sim 10 \mathrm{~Hz})$ oscillation is more consistent with the alpha band of the EEG spectrum. Moreover, a period doubling cascade is supported and beyond this the model is known to support chaos. Indeed the chaotic behaviour has been extensively investigated in a series of papers [31,32,30], highlighting that the route to chaos is actually via a novel Shilnikov saddle-node bifurcation. Note that in contrast the chaotic EEG patterns in the Freeman model of the olfactory system are generated via the Ruelle-Takens-Newhouse route [33]. For further discussion on the use of neural mass models in epileptic modelling and human brain rhythms we refer the reader to [34,35] and [36] respectively.

It is possible to recover a solely activity based model from the Liley model by treating the limit of fast relaxation $\tau_{a} \rightarrow 0$. In this case the mean membrane voltages are slaved to the dynamically evolving con-

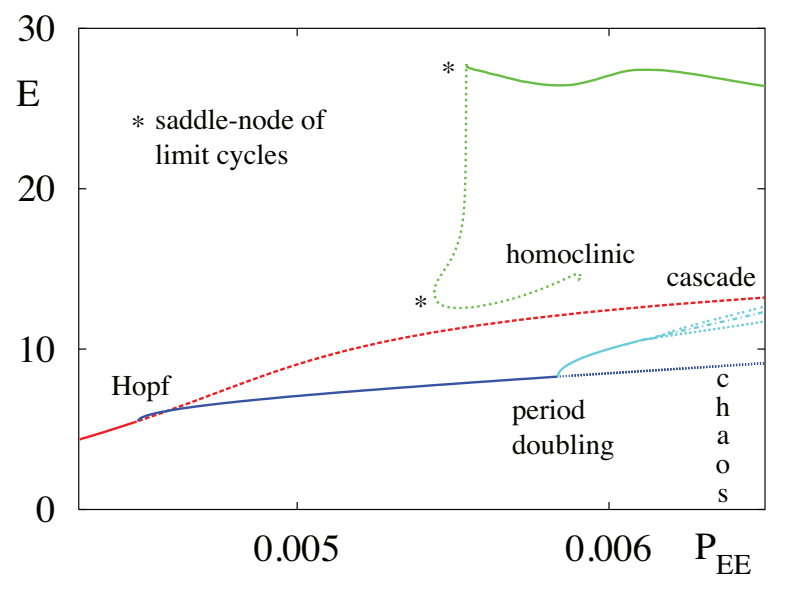

Figure 2: Bifurcation diagram for the Liley population model showing the absolute maximum of $E$ in terms of $P_{E E}$ for steady state (red), small amplitude periodic (blue) and large amplitude periodic (green) orbits. Stable (unstable) branches are solid (dashed). Chaotic solutions are found after the period doubling cascade. Parameters are modified from [30] as $P_{I E}=0.005763$ $\mathrm{ms}^{-1}, \alpha_{E E}=\alpha_{I E}=1.01 \mathrm{~ms}^{-1}, \alpha_{I I}=\alpha_{E I}=0.142$ $\mathrm{ms}^{-1}, W_{E E}=W_{I E}=43.31, W_{I I}=W_{E I}=925.80$, $\beta_{E}=0.3, \beta_{I}=0.27, \theta_{E}=21.0 \mathrm{mV}, \theta_{I}=29.0 \mathrm{mV} h_{E}=$ $115.0 \mathrm{mV}, h_{I}=-20.0 \mathrm{mV}$ with zero-delays $\Delta_{a b}=0$.

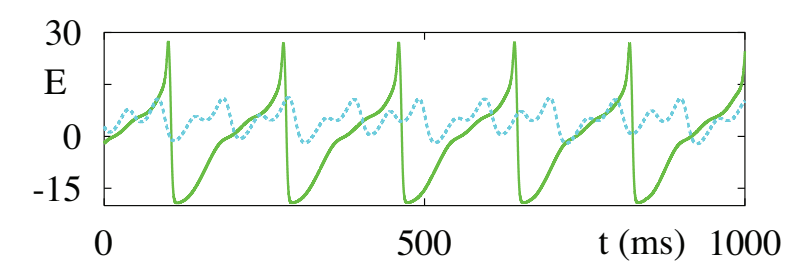

Figure 3: An example of multistability in the Liley population model. A small and large amplitude stable rhythm co-exist over a range of parameter values. Here parameters are as in Fig. 2 with $P_{E E}=0.006216$ $\mathrm{ms}^{-1}$. 
ductances and we may write $E=E\left(g_{E E}, g_{E I}\right)$ and $I=I\left(g_{I I}, g_{I E}\right)$. Symbolically the model now takes the closed form

$$
g=\eta *[f+P], \quad f=f(\{g\}),
$$

where we suppress indices and use the notation $\{g\}$ to emphasise that the firing rate depends on the set of network conductances. A whole host of models can be described with such a system of equations, ranging from networks with many more than two modules and a complicated dependence of the firing rate on the set of network conductances down to a single population with self-excitation. A very simple example of this latter case for a single conductance $g=u$ can be obtained for the choice of an exponential synapse $\eta(t)=\alpha \mathrm{e}^{-\alpha t} H(t)$ and assuming $f=f(u)$ with $P=0$, so that

$$
\left(1+\frac{1}{\alpha} \frac{\mathrm{d}}{\mathrm{d} t}\right) u=f(u) .
$$

In some sense we may regard this model as one of the most basic to arise in mathematical neuroscience. Interestingly it has been subjected to modifications that improve its ability to model neuronal dynamics without recourse to abandoning the fast relaxation assumption. One of the main extensions of this type has been the inclusion of a term to describe refractoriness [2]. A particular example is that of Curtu and Ermentrout [37]:

$$
\frac{1}{\alpha} \frac{\mathrm{d} u}{\mathrm{~d} t}=-u+\left(1-\frac{1}{R} \int_{t-R}^{t} u(s) \mathrm{d} s\right) f(u),
$$

where $R$ is the absolute refractory period of the neurons in the population. The fixed point $u_{\mathrm{ss}}$ satisfies the equation $-u_{\mathrm{ss}}+\left(1-u_{\mathrm{ss}}\right) f\left(u_{\mathrm{ss}}\right)=0$. For a sigmoid with $0<f<1$ then there is at least one solution of this equation for $u_{\mathrm{ss}} \in(0,1 / 2)$. The characteristic equation determining the linear stability of the steady state is calculated as $\mathcal{E}(\lambda)=0$, with

$$
\mathcal{E}(\lambda)=\frac{\lambda}{\alpha}+A+f\left(u_{\mathrm{ss}}\right) \frac{1-\mathrm{e}^{-\lambda R}}{\lambda R},
$$

where $A=1-\left(1-u_{\mathrm{ss}}\right) f^{\prime}\left(u_{\mathrm{ss}}\right)$. This transcendental equation allows for the possibility of complex roots, and not surprisingly it is possible to choose values for the slope and threshold of the sigmoid such that

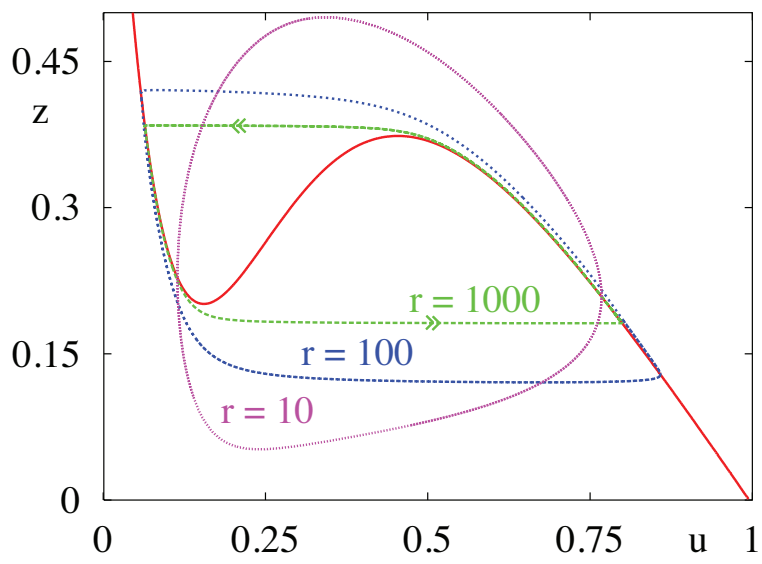

Figure 4: Relaxation oscillations in an activity based single population with self-excitation and refractoriness. $r=1000, T \sim 1.29 . r=100, T \sim 1.43 . r=10, T \sim$ 2.24. $\beta=8, \theta=1 / 3$. The cubic curve is $z=1-u / f(u)$.

a dynamic bifurcation defined by $\mathcal{E}(i \omega)=0$ can occur [37]. Interestingly periodic solutions that emerge beyond such a bifurcation can be analysed explicitly in some singular limit. To see this we write (11) as a twodimensional delay differential equation:

$$
\begin{aligned}
\epsilon \dot{u} & =-u+(1-z) f(u), \\
\dot{z} & =u(t)-u(t-1),
\end{aligned}
$$

where we have re-scaled time according to $t \mapsto t / R$ and set $\epsilon=(\alpha R)^{-1}$. Formally setting $\epsilon=0$ gives the graph $z=1-u / f(u)$, which we show an example of in Fig. 4. Note the 'cubic' shape of this curve, reminiscent of the nullcline for the fast variable seen in many excitable systems, and particularly those of FitzHugh-Nagumo type [38]. In the limit $\epsilon \rightarrow 0$ dynamics consists of slow evolution along the left and right branches of the 'cubic' with fast transitions from one branch to another. In this case it can be shown that the period of oscillation satisfies $T<2 R$ (and numerical experiments show that the actual oscillation period scales linearly with $R$ ). Hence, the inclusion of refractoriness in activity based models is a natural way to induce oscillations with an emergent period that is largely set by the refractory time-scale. Another extension of the basic model (10) is to recognise that in cortical tissue there are an abundance of metabolic 
processes whose combined effect is to modulate neuronal response. It is convenient to think of these processes in terms of local feedback mechanisms. For example, spike frequency adaptation (SFA) is a property of many single neurons and has been linked to the presence of a $\mathrm{Ca}^{2+}$ gated $K^{+}$current, $I_{\mathrm{AHP}}$ [39]. The generation of an action potential leads to a small calcium influx that increments $I_{\mathrm{AHP}}$, with the end result being a decrease in the firing rate response to persistent stimuli. A simple phenomenological model of this process is to add a current, that activates in the presence of high activity, $I-g s$, to the right hand side of (10) [40], where

$$
\tau \frac{\mathrm{d} s}{\mathrm{~d} t}=-s+f(u)
$$

and $I$ is a constant bias. This form of SFA leads naturally to the emergence of periodic behaviour. Indeed in the limit of large $\tau$ (slow adaptation) and a for a steep sigmoid (large $\beta$ ) this period can be calculated explicitly as $\tau \log ((g-I)(1+I) /(I(g-1-I))$ for $g>1+I$ and shows a classic relaxation oscillation between up and down states like those seen in biophysical models of slow $(<1 \mathrm{~Hz})$ oscillations [41]. An alternative approach, consistent with observations first made by Hill in 1936 [42], is to treat the threshold in the firing function to be state-dependent. This is explored in detail in $[43,44]$ and shown to lead to exotic dynamics at the network level, including the emergence of dissipative solitons. However, rather than pursue these extensions in more detail we shall instead next show how to build tissue level models taking as a starting point models of the form (9).

\section{Tissue models}

Here we will view a macroscopic part of the neocortex as being adequately modelled as a spatial assembly of population models - a neural field model. Such a viewpoint has already proven useful in understanding spatial aspects of the alpha rhythm and in particular cortical travelling waves $[6,45]$. For a recent perspective on the use of neural field models in interpreting extrinsic optical imaging data (from in vitro exper- iments on pharmacologically treated brain slices) we refer the reader to [46].

To develop the extension of (9) to treat spatially continuous neural sheets (such as a two-dimensional cortex) we will adopt the continuum assumption and treat a density of neurons at a point with inputs that arise from the delayed and weighted contribution of activity at other points in the tissue. Because these interactions are mediated by long-range axonal fibres the resulting tissue-level model is inherently non-local and is often cast in the form of an integral equation. We represent this symbolically in the form

$$
g=w \otimes \eta * f,
$$

where the operator $\otimes$ captures information about both anatomical connectivity patterns and the distribution of axonal delays. As a concrete example consider a set of two-dimensional interacting layered sheets (with both self and layer to layer interactions), each containing only one cell type (either excitatory or inhibitory). The activity in layer $a$ induced by that in layer $b$ (generalising equation (9)) then takes the form

$$
u_{a b}=\eta_{a b} * \psi_{a b},
$$

where $\psi_{a b}=\psi_{a b}(\mathbf{r}, t)$ is given by

$$
\int_{\mathbb{R}^{2}} \mathrm{~d} \mathbf{r}^{\prime} w_{a b}\left(\mathbf{r}, \mathbf{r}^{\prime}\right) f_{b}\left(\mathbf{r}^{\prime}, t-\left|\mathbf{r}-\mathbf{r}^{\prime}\right| / v_{a b}\right)
$$

and $f_{b}$ is the firing rate in layer $b$. Here $\mathbf{r} \in \mathbb{R}^{2}$ and $w_{a b}\left(\mathbf{r}, \mathbf{r}^{\prime}\right)$ prescribes the coupling strength between position $\mathbf{r}$ in layer $a$ and position $\mathbf{r}^{\prime}$ in layer $b$. The velocity of an action potential travelling along a fibre connecting layer $b$ to layer $a$ is denoted $v_{a b}$ and underlies the space-dependent delay $\left|\mathbf{r}-\mathbf{r}^{\prime}\right| / v_{a b}$ for signals propagating over a distance $\left|\mathbf{r}-\mathbf{r}^{\prime}\right|$. Note that (18) provides meaning for the operator on the right hand side of the expression $\psi=w \otimes f$. To close the system of equations one could choose the firing rate to depend on some dynamic mean-membrane potential as in the Liley model. Alternatively, to recover a purely activity based model in the spirit of Wilson-Cowan and Amari one could set $f_{a}=f_{a}\left(h_{a}\right)$, where $h_{a}=\sum_{b} u_{a b}$. For simplicity we shall restrict further discussion to this case. For a recent historical perspective of the WilsonCowan model we refer the reader to [47]. Suffice to 
say that, apart from their relevance to neuroimaging, neural field models have found many applications in neuroscience, including to understanding the generation of visual hallucinations [48, 49], modelling orientation tuning in visual cortex area v1 [10], describing travelling waves of activity in v1 during binocular rivalry [50,51], models of working memory [7] and encoding of continuous stimuli [52], motion perception [8], somatosensory illusions [53], and developing a theory of cognitive robotics [54] for example.

Neural field models of the type (16) are nonlinear spatially extended systems and thus have all the necessary ingredients to support pattern formation. The analysis of such behaviour is typically performed with a mixture of linear Turing instability theory, weakly nonlinear perturbative analysis and numerical simulations (see [55] for a review). In the absence of detailed anatomical data it is common practice to consider cortico-cortical connectivity functions to be homogeneous and isotropic so that $w_{a b}\left(\mathbf{r}, \mathbf{r}^{\prime}\right)=w_{a b}(\mid \mathbf{r}-$ $\left.\mathbf{r}^{\prime} \mid\right)$. In this case a homogeneous steady state is expected and can be defined by $h_{a}^{\mathrm{ss}}=\sum_{b} W_{a b} f_{b}\left(h_{b}^{\mathrm{ss}}\right)$, where $W_{a b}=\int_{\mathbb{R}^{2}} \mathrm{~d} \mathbf{r} w_{a b}(\mathbf{r})$. For concreteness we shall take

$$
w_{a b}(r)=w_{a b}^{0} \mathrm{e}^{-r / \sigma_{a b}} /(2 \pi),
$$

where $r=|\mathbf{r}|$. Linearising around the steady state and considering perturbations of the form $h_{a}(\mathbf{r}, t) \sim$ $\mathrm{e}^{\lambda t} \mathrm{e}^{i \mathbf{k} \cdot \mathbf{r}}$, gives an equation for the continuous spectrum $\lambda=\lambda(k)$, for $k=|\mathbf{k}|$, in the form $\mathcal{E}(k, \lambda)=0$ [56], where $\mathcal{E}(k, \lambda)=\operatorname{det}(\mathcal{D}(k, \lambda)-I)$, and

$$
[\mathcal{D}(k, \lambda)]_{a b}=\widetilde{\eta}_{a b}(\lambda) G_{a b}(k,-i \lambda) \gamma_{b} .
$$

Here $\gamma_{a}=f_{a}^{\prime}\left(h_{a}^{\mathrm{ss}}\right)$ and $G_{a b}(k, \omega)$ is the Fourier transform of $G_{a b}(r, t)=w_{a b}(r) \delta\left(t-r / v_{a b}\right)$ defined by

$$
\begin{aligned}
G_{a b}(k, \omega) & =\int_{\mathbb{R}^{3}} \mathrm{~d} \mathbf{r} \mathrm{d} t G_{a b}(r, t) \mathrm{e}^{-i(\mathbf{k} \cdot \mathbf{r}+\omega t)} \\
& =w_{a b}^{0} \frac{A_{a b}(\omega)}{\left(A_{a b}^{2}(\omega)+k^{2}\right)^{3 / 2}}
\end{aligned}
$$

where $A_{a b}(\omega)=1 / \sigma_{a b}+i \omega / v_{a b}$. Here we use a notation that distinguishes functions and their transforms simply by their arguments, namely $(r, t)$ for the original space and $(k, \omega)$ for the Fourier space. An instability occurs when for the first time there are values of $k$ at which the real part of $\lambda$ is non-negative.

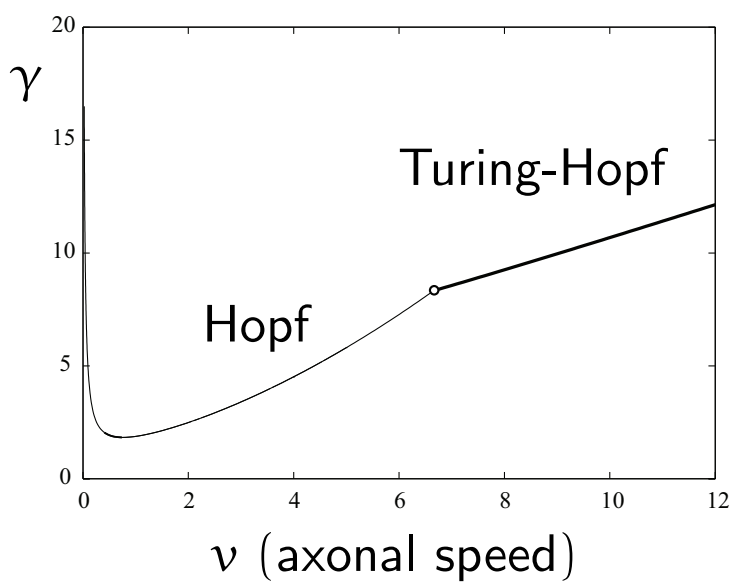

Figure 5: Critical curves showing the instability borders for dynamic instabilities in the $(v, \gamma)$ plane, where $\gamma=f^{\prime}\left(h^{\mathrm{ss}}\right)$.

A Turing bifurcation point is defined as the smallest value of some order parameter for which there exists some non-zero $\mathbf{k}_{c}$ satisfying $\operatorname{Re}\left(\lambda\left(\left|\mathbf{k}_{c}\right|\right)\right)=0$. It is said to be static if $\operatorname{Im}\left(\lambda\left(\left|\mathbf{k}_{c}\right|\right)\right)=0$ and dynamic if $\operatorname{Im}\left(\lambda\left(\left|\mathbf{k}_{c}\right|\right)\right) \equiv \omega_{c} \neq 0$. The dynamic instability is often referred to as a Turing-Hopf bifurcation and generates a global pattern with wavenumber $\left|\mathbf{k}_{c}\right|$, which moves coherently with a speed $c=\omega_{c} /\left|\mathbf{k}_{c}\right|$, i.e. as a periodic travelling wave train. If the maximum of the dispersion curve is at $\left|\mathbf{k}_{c}\right|=0$ then the mode that is first excited is another spatially uniform state. If $\omega_{c} \neq 0$, we would then expect the emergence of a coherent network oscillation with frequency $\omega_{c}$.

For example, consider two populations, one excitatory and one inhibitory with a common firing rate function $f_{a}=f$ and single axonal conduction velocity $v_{a b}=v$, and use the labels $a \in\{E, I\}$, with $w_{E E, I E}^{0}=1$, $w_{I I, E I}^{0}=-4, \alpha_{a b}=1$ and $\Delta_{b a}=0$. In neocortex the extent of excitatory connections $W_{a E}$ is broader than that of inhibitory connections $W_{a I}$, and so we take $\sigma_{a I}=1$ and $\sigma_{a E}=2$. In Fig. 5 we show a plot of the critical curves in the $(v, \gamma)$ plane above which the homogeneous steady state, $h_{E, I}^{\mathrm{ss}}=h^{\mathrm{ss}}$, is unstable to dynamic instabilities with $\left|\mathbf{k}_{c}\right|=0$ (bulk oscillations) and $\left|\mathbf{k}_{c}\right| \neq 0$ (travelling waves). Figure 6 shows a pattern of parallel moving stripes seen beyond the Turing-Hopf 


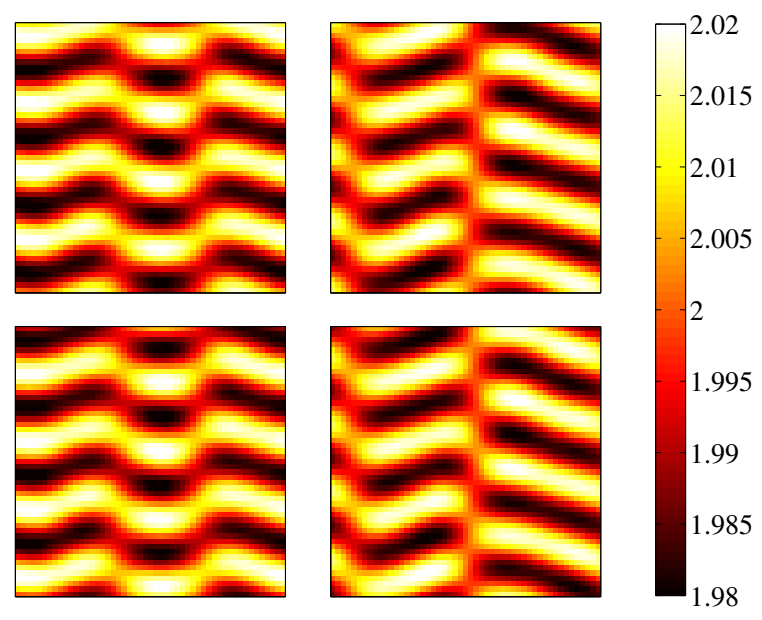

Figure 6: Snapshots of a periodic travelling wave in $u_{E E}$, each $1 / 4$ of a period later than the previous one (ordered top left, top right, bottom left, bottom tight), for the 2D model of Fig. 5 at $v=12$ and $\gamma=15$ (on a $30 \times 30$ domain).

\section{bifurcation.}

Despite the natural framework for neural field models being non-local integral equations with spacedependent delays the techniques for analysing them are nowhere near as developed as they are for local PDE models. Thus for this reason it is sometimes worth constructing equivalent PDE models. To date progress in this area has been made by Nunez [6] and Jirsa and Haken [19] for neural field models in one spatial dimension with axonal delays, making links to the theory of damped inhomogeneous wave equations. For example in a one-dimensional setting with $w_{a b}(x)=\mathrm{e}^{-|x| / \sigma_{a b}} /\left(2 \sigma_{a b}\right)$, the equivalent PDE model to $\psi=w \otimes f$ is

$$
\left[\mathcal{A}_{a b}^{2}-\partial_{x x}\right] \psi_{a b}=\frac{1}{\sigma_{a b}} \mathcal{A}_{a b} f_{b},
$$

where

$$
\mathcal{A}_{a b}=\left(\frac{1}{\sigma_{a b}}+\frac{1}{v_{a b}} \partial_{t}\right) .
$$

Equation (22) is the Jirsa-Haken-Nunez brain wave equation $[19,6$, 57]. Turing instabilities in such models (built from two populations) have been exhaustively analysed in $[58,59]$ and lead to bifurcation scenarios consistent with those in Fig. 5, when considering analogous architectures. Moreover, a weakly non- linear analysis of the travelling and standing waves that develop beyond the point of instability has been developed. The appropriate amplitude equations are found to be the coupled mean-field Ginzburg-Landau equations describing a Turing-Hopf bifurcation with modulation group velocity of $O(1)$. In particular this has allowed an investigation of Benjamin-Feir modulational instabilities in which a periodic travelling wave (of moderate amplitude) loses energy to a small perturbation of other waves with nearly the same frequency and direction. For asymmetric kernels $(w(x) \neq$ $w(-x))$ or symmetric kernels with a peak away from the origin then the stability analysis of the homogeneous steady state involves the solution of transcendental equations (as it would for an ordinary differential equations with a fixed delay), though can be analysed making use of Lambert functions [60].

For a single self-coupled population with $\sigma_{a a}=\sigma$, $v_{a a}=v$ and a simple exponential synaptic filter $\eta_{a a}=$ $\alpha \mathrm{e}^{-\alpha t} H(t)$, (22) also supports travelling fronts [61]. Moreover, for steep sigmoids $\left(\beta \rightarrow \infty\right.$ and $\theta_{a}=\theta$ ) the speed can be calculated in closed form as

$$
c=\frac{(2 \theta-1) v}{2 \theta-1-2 \theta v /(\alpha \sigma)} .
$$

This strong dependence of the wave speed on the threshold $\theta$ has now been indirectly established in rat cortical slices (bathed in the $\mathrm{GABA}_{\mathrm{A}}$ blocker picrotoxin) [62]. An applied positive (negative) electric field across the slice increased (decreased) the speed of wave propagation, consistent with (24) assuming that a positive (negative) electric field reduces (increases) the threshold $\theta$. Of course travelling waves are also possible solutions of the more general non-local formalism described by (16), and have been explored mathematically in a number of papers, reviewed in [11], with a particular regard to studying waves in cortical slices [63], and notably epileptiform activity [64].

As well as supporting periodic travelling waves the brain wave equation (22) can support propagating pulses. A detailed mathematical analysis of waves of this type has been performed in [65] for the case of a Heaviside firing rate function (a sigmoid with infinite gain). The treatment of smooth firing rates can be pur- 


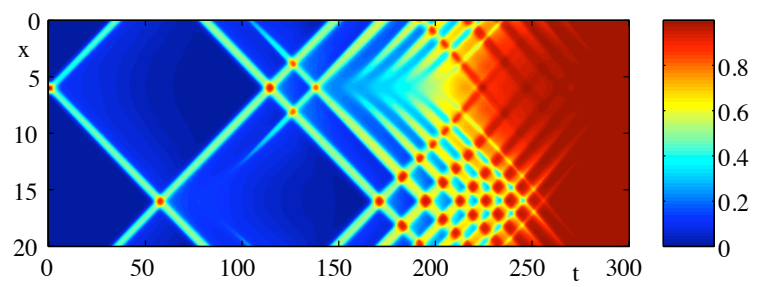

Figure 7: Numerical simulations of a coupled neural field model in one spatial dimension showing the interaction of two travelling pulses and the emergence of transient complex behaviour ultimately leading to an elevated firing rate across the whole tissue. The model is defined by $\left[\mathcal{A}_{a}^{2}-\partial_{x x}\right] \psi_{a}=\Gamma_{a} \mathcal{A}_{a} H\left(u_{e}-u_{i}-\right.$ $\theta) / \sigma_{a}, \mathcal{A}_{a}=\left(\sigma_{a}^{-1}+v_{a}^{-1} \partial_{t}\right),\left(1+\alpha_{a}^{-1} \partial_{t}\right) u_{a}=\psi_{a}$, with $a \in\{e, i\}$, describing a model with short range inhibition and long range excitation. The plot shows the evolution of $u_{e}$ with $v_{e}=0.2, v_{i}=1, \alpha_{e}=\alpha_{i}=1$, $\Gamma_{e}=1, \Gamma_{i}=0.7, \sigma_{e}=2, \sigma_{i}=1$ and $\theta=0.07$.

sued for waves in one space-dimension using a standard travelling wave analysis (in which one looks for stationary profiles in a co-moving frame $\xi=x-c t$ of speed $c<v$ ). This can often only be done numerically, say using the techniques of spatial dynamics and constructing homoclinic connections, as in [61]. Interestingly propagating pulses of (22) can scatter in novel ways, leading to an elevated firing across the whole tissue [65]. An example of this is shown in Fig. 7.

Finding equivalent brain wave equations in twospatial dimensions has proved far more challenging [18], with various approximations being made to obtain a local model. The most common of these is the so-called long-wavelength approximation, although this has recently been improved upon in [56]. In the former case this amounts to expanding (21) around $k=0$ for small $k$, yielding a "nice" rational polynomial structure to give $\left(A_{a b}^{2}(\omega)+3 k^{2} / 2\right) \psi_{a b}(k, \omega)=$ $f_{b}(k, \omega)$ which may then be inverse transformed to give the telegraph PDE:

$$
\left(\mathcal{A}_{a b}^{2}-\frac{3}{2} \nabla^{2}\right) \psi_{a b}=w_{a b}^{0} f_{b} .
$$

This model has been intensively studied by a number of authors in the context of EEG modelling, see for example $[66,67,68]$.
Undoubtedly the assumption of isotropic connectivity is a strong one for the modelling of cortical tissue. That it has been pursued so aggressively to date is more a reflection of the mathematical tractability of such models as opposed to their relation to real tissue. Indeed questions about the existence, uniqueness and absolute stability of solutions for truly inhomogeneous models are only just beginning to be addressed using tools from functional analysis [69]. However, one symmetry breaking effect that can be tackled without too much effort is that of the loss of continuous rotation symmetry. This is an important issue to treat in light of the fact that it is now known that (visual) cortex has a crystalline micro-structure at the millimeter length scale (reviewed in [70]). This has given rise to the notion of patchy connections that break continuous rotation symmetry (but not necessarily continuous translation symmetry). The natural way to model this is to introduce a modified connectivity kernel $w_{a b}^{\mathrm{P}}\left(\mathbf{r}, \mathbf{r}^{\prime}\right)$ as

$$
w_{a b}^{\mathrm{P}}\left(\mathbf{r}, \mathbf{r}^{\prime}\right)=w_{a b}\left(\left|\mathbf{r}-\mathbf{r}^{\prime}\right|\right) J_{a b}\left(\mathbf{r}-\mathbf{r}^{\prime}\right),
$$

where $J_{a b}(\mathbf{r})$ varies periodically with respect to a regular planar lattice $\mathcal{L}$. Note that the patchy kernel $w_{a b}^{P}$ is homogeneous, but not isotropic. The generalisation of brain wave equations to treat patchiness that arise via periodic modulation of an isotropic kernel has recently been developed by Robinson [71] and analysed further in [56]. In essence the brain wave equation (25) is replaced by an infinite set of PDEs - indexed by the reciprocal lattice vectors $\mathbf{q}$ of the underlying lattice $\mathcal{L}$, that arise in the Fourier series representation

$$
J_{a b}(\mathbf{r})=\sum_{\mathbf{q}} J_{a b}^{\mathbf{q}} \mathrm{e}^{i \mathbf{q} \cdot \mathbf{r}} .
$$

In the long-wavelength approximation this set of PDEs is obtained from (25) under the replacement $\nabla \rightarrow \nabla-i \mathbf{q}, \psi_{a b} \rightarrow \psi_{a b}^{\mathbf{q}}$ and $u_{a b} \rightarrow \eta_{a b} * \sum_{\mathbf{q}} J_{a b}^{\mathbf{q}} \psi_{a b}^{\mathbf{q}}$. Assuming that there is a natural cut-off in $\mathbf{q}$, then we need only evolve a finite subset of these PDEs to see the effects of patchy connections on solution behaviour. For example with a square lattice we would need only to specify two reciprocal lattice vectors. In this case a Turing instability analysis shows that, 
compared to the unmodulated case shown in Fig. 5, the Hopf bifurcation is transformed to a Turing-Hopf bifurcation with critical wavevectors coinciding with those of the lattice. With increasing $v$ the dominant bifurcation is also of Turing-Hopf type. However, in this case it is a ring of wavevectors surrounding the reciprocal lattice vectors that go unstable first. In both cases this suggests the emergence of periodic travelling waves aligned to the lattice size and direction, which are indeed observed in direct numerical simulations [56]. For a more general inhomogeneous kernel of the form $w_{a b}^{\mathrm{I}}\left(\mathbf{r}, \mathbf{r}^{\prime}\right)=w_{a b}\left(\left|\mathbf{r}-\mathbf{r}^{\prime}\right|\right) J_{a b}\left(\mathbf{r}^{\prime}\right)$, then the PDE formulation goes over with the source term $f_{b}(\mathbf{r}, t)$ in (25) replaced by $J_{a b}(\mathbf{r}) f_{b}(\mathbf{r}, t)$. When the modulating kernel $J_{a b}(\mathbf{r})$ varies both weakly and rapidly in space then one may use techniques from homogenisation theory to study wave propagation and its failure [72]. Indeed recent work avoiding the assumption of rapid spatial variation shows that for a one dimensional neural field model with $J(x)=1+\epsilon \sin (2 \pi x / \sigma)$ and $f(u)=H(u-\theta)$ then pulsating waves can occur (where say a front edge is modulated in space) with a wave speed $c_{0} \sqrt{1-\left(\epsilon / \epsilon_{c}\right)^{2}}$ so that propagation failure occurs for some $\epsilon>\epsilon_{c}=\epsilon_{c}(\sigma, \theta)$ [73]. Here $c_{0}$ is the speed of the wave in the homogeneous case (when $\epsilon=0$ and see equation (24) for example). Further treatment of heterogeneous connection topologies can be found in $[74,75,76,77]$.

One of the more recent and compelling uses of neural field modelling is by Bojak and colleagues [13, 14]. They relate different (co-registered) imaging modalities to one another, namely EEG (with its excellent temporal resolution) and fMRI (with its superior spatial resolution) by modelling an underlying neural generator that is based upon the Liley model [18] discussed earlier. Importantly regional connectivity data is incorporated using the CoCoMac database [78], which contains information on structural connectivity in the macaque brain (from tract-tracing experiments). In order to be compatible with true fMRI images their computational model uses triangular spatial grids matched to cortical geometries extracted from structural MR images. From the activity of the

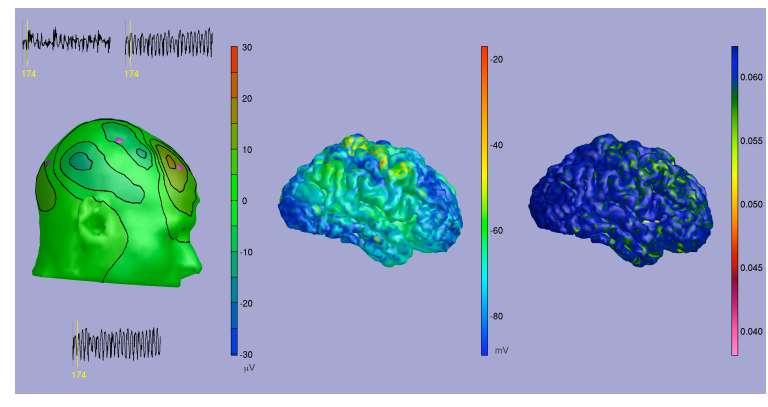

Figure 8: An EEG scalp isopotential (left) generated from the mean membrane excitatory potential of a neural field model (middle) and the corresponding fMRI BOLD signal construction on the cortical surface (right), illustrating the capabilities of the recent computational framework developed by Bojak and colleagues $[13,14]$. Associated animations may be found at http://www.mbfys.ru.nl/neuropi/cns09/.

model cortical sheet, the simulated EEG at the scalp is found using a realistic volume conductor model. The response for fMRI BOLD is constructed using an established model for neurovascular coupling and convolving with "Balloon-Windkessel" haemodynamics [79]. This is one of the first attempts to seriously combine neuronal dynamics and brain connectivity along the lines advocated in [80]. An example of output from their modelling approach is shown in Fig. 8. For a recent review on the challenges in combining EEG and fMRI imaging modalities using neural models we refer the reader to Valdes-Sosa et al. [81], and for work on folded three-dimensional cortical sheets and their use in forward EEG and MEG prediction see Jirsa et al. [82]. Other work on using realistic anatomical connectivities in large scale neural models (especially as it relates to the resting brain state) can be found in [83, 84, 85].

There are a number of natural extensions to neural field models to incorporate ever more biological realism, including the incorporation of slow intrinsic currents that underlie bursting behaviour in single neurons [86, 11], synaptic depression and adaptation [87], dendritic processing [61], neuromodulation, say by endo-cannabinoids [88], and anaesthetic drug action $[66,89,68,90]$. Moreover, it is possible to gener- 
alise such models even further to treat feature selectivity such as that observed in visual cortex for orientation [10], spatial frequency [91] and texture [92]. In this case neural activity is now also considered to be a function of some set of features $\chi$ and interactions must be specified by a feature dependent kernel $w_{a b}\left(\mathbf{r}^{\prime}, t^{\prime}, \chi^{\prime} \mid \mathbf{r}, t, \chi\right)$. Indeed with the increasing march of experimental progress in recording from populations of neurons we are now ideally poised to push forward with the development of multi-scale models that can integrate macroscopic models at large spatial scales with models at the microscopic scale [93]. One outstanding challenge for the development of tissue level firing rate models is how best to include gap junction coupling [94].

\section{Discussion}

A known limitation of neural field models is that they only try to track mean activity levels and cannot, by definition, track the higher order correlations of any underlying spiking model. Of course one approach would be to abandon them altogether in favour of more biophysically detailed models. However, as we have discussed here they can go a remarkably long way to providing a framework for interpreting neuro-imaging data whilst maintaining contact with known brain structure, dynamics and function. Rather it is preferable to work with spiking models and establish more concretely the link to mean activity models, and when more sophisticated kinetic models of brain activity are required [95, 96]. Moreover, in some instances it is possible to analyse spiking networks directly (usually under the assumption of global coupling and fast synaptic interactions) as in the spike-density approach [97, 98, 99], which makes heavy use of the numerical solution of coupled partial differential-integral equations. In other situations equations going beyond the mean-field approach have been proposed that govern second-order correlations [100, 101, 102, 103]. Indeed there has been a recent upsurge of interest in this area adapting methods from non-equilibrium statistical physics to determine corrections to mean-field theory involving equations for two-point and higher-order cumulants $[104,105]$. One immediate, yet potentially tractable, challenge would be to develop a framework for understanding networks of synaptically interacting nonlinear integrate-and-fire networks. At the single neuron level such models are already known to be able to capture many different physiological firing patterns and at the network level are computationally far cheaper to implement than their conductance based cousins yet still able to generate the rich repertoire of behaviour seen in a real nervous system [106]. One step in this direction has already been undertaken for the absolute-integrate-and-fire model [107] and this may well be a natural spiking model to pursue for the development of a specific soluble spiking neurodynamics.

\section{Acknowledgements}

SC would like to thank Carlo Laing and David Liley for many interesting discussions concerning the modelling of cortical tissue and Ingo Bojak for providing Fig. 8.

\section{References}

[1] H Markram. The blue brain project. Nature Reviews Neuroscience, 7:153-160, 2006.

[2] H R Wilson and J D Cowan. Excitatory and inhibitory interactions in localized populations of model neurons. Biophysical Journal, 12:1-24, 1972.

[3] H R Wilson and J D Cowan. A mathematical theory of the functional dynamics of cortical and thalamic nervous tissue. Kybernetik, 13:55-80, 1973.

[4] S Amari. Homogeneous nets of neuron-like elements. Biological Cybernetics, 17:211-220, 1975.

[5] S Amari. Dynamics of pattern formation in lateralinhibition type neural fields. Biological Cybernetics, 27:77-87, 1977.

[6] P L Nunez. The brain wave equation: a model for the EEG. Mathematical Biosciences, 21:279-297, 1974. 
[7] C R Laing, W C Troy, B Gutkin, and G B Ermentrout. Multiple bumps in a neuronal model of working memory. SIAM Journal on Applied Mathematics, 63:62-97, 2002.

[8] M A Geise. Neural Field Theory for Motion Perception. Kluwer Academic Publishers, 1999.

[9] K Zhang. Representation of spatial orientation by the intrinsic dynamics of the head-direction cell ensemble: A theory. Journal of Neuroscience, 16:2112-2126, 1996.

[10] R Ben-Yishai, L Bar-Or, and H Sompolinsky. Theory of orientation tuning in visual cortex. Proceedings of the National Academy of Sciences USA, 92:3844-3848, 1995.

[11] S Coombes. Waves, bumps, and patterns in neural field theories. Biological Cybernetics, 93:91-108, 2005.

[12] S Coombes. Neural fields. Scholarpedia: http://www.scholarpedia.org /article/Neural_fields, 1:1373, 2006.

[13] I Bojak, T F Oostendorp, A T Reid, and R Kötter. Realistic mean field forward predictions for the integration of co-registered EEG/fMRI. BMC Neuroscience, 10:L2, 2009.

[14] T F Oostendorp, I Bojak, A T Reid, and R Kötter. Connecting mean field models of neural activity to EEG and fMRI data. Brain Topography, to appear, 2009.

[15] P beim Graben and R Potthast. Inverse problems in dynamic cognitive modeling. Chaos, 19:015103, 2009.

[16] K E Stephan, L M Harrison, S J Kiebel, O David, W D Penny, and K J Friston. Dynamic causal models of neural system dynamics: current state and future extensions. Journal of Biosciences, 32:129-144, 2007.

[17] J Daunizeau, S J Kiebel, and K J Friston. Dynamic causal modelling of distributed electromagnetic responses. NeuroImage, 47:590-601, 2009.

[18] D T J Liley, P J Cadusch, and M P Dafilis. A spatially continuous mean field theory of electrocortical activity. Network, 13:67-113, 2002.

[19] V K Jirsa and H Haken. A derivation of a macroscopic field theory of the brain from the quasi-microscopic neural dynamics. Physica D, 99:503-526, 1997.

[20] B H Jansen and V G Rit. Electroencephalogram and visual evoked potential generation in a mathematical model of coupled cortical columns. Biological Cybernetics, 73:357-366, 1995.
[21] F H Lopes da Silva, A Hoeks, and L H Zetterberg. Model of brain rhythmic activity. Kybernetik, 15:27-37, 1974.

[22] O David and K J Friston. A neural mass model for MEG/EEG: coupling and neuronal dynamics. NeuroImage, 20:1743-1755, 2003.

[23] F Grimbert and O Faugeras. Bifurcation analysis of Jansen's neural mass model. Neural Computation, 18:3052-3068, 2006.

[24] R A Stefanescu and V K Jirsa. A low dimensional description of globally coupled heterogeneous neural networks of excitatory and inhibitory neurons. PLoS Compututational Biology, 4:e1000219, 2008.

[25] C R Laing. On the application of "equation-free modelling" to neural systems. Journal of Computational Neuroscience, 20:5-23, 2006.

[26] C R Laing and I G Kevrekidis. Periodically-forced finite networks of heterogeneous coupled oscillators: a low-dimensional approach. Physica D, 237:207-215, 2008.

[27] S Coombes and C Laing. Delays in activity-based neural networks. Philosophical Transactions of the Royal Society A, 367:1117-1129, 2009.

[28] M Breakspear, J A Roberts, J R Terry, S Rodrigues, $\mathrm{N}$ Mahant, and P A Robinson. A unifying explanation of primary generalized seizures through nonlinear brain modeling and bifurcation analysis. Cerebral Cortex, 16:1296-1313, 2006.

[29] B Ermentrout. Simulating, Analyzing, and Animating Dynamical Systems: A Guide to XPPAUT for Researchers and Students. Society for Industrial \& Applied Mathematics, 2002.

[30] M P Dafilis, F Frascoli, P J Cadusch, and D T J Liley. Chaos and generalised multistability in a mesoscopic model of the electroencephalogram. Physica D, 238:1056-1060, 2009.

[31] M P Dafilis, D T J Liley, and P J Cadusch. Robust chaos in a model of the electroencephalogram: Implications for brain dynamics. Chaos, 11:474-478, 2001.

[32] L van Veen and D T J Liley. Chaos via Shilnikov's saddle-node bifurcation in a theory of the electroencephalogram. Physical Review Letters, 97:208101, 2006.

[33] W J Freeman. Simulation of chaotic EEG patterns with a dynamic model of the olfactory system. Biological Cybernetics, 56:139-150, 1987. 
[34] J Milton and P Jung, editors. Epilepsy as a Dynamic Disease. Springer, 2003.

[35] B Schelter and J Timmer, editors. Seizure Prediction in Epilepsy: From Basic Mechanisms to Clinical Applications. Wiley-VCH, 2008.

[36] D A Steyn-Ross and M Steyn-Ross, editors. Modeling Phase Transitions in the Brain, volume 4 of Springer Series in Computational Neuroscience. Springer, 2010.

[37] R Curtu and B Ermentrout. Oscillations in a refractory neural net. Journal of Mathematical Biology, 43:81-100, 2001.

[38] R Fitzhugh. Impulses and physiological states in theoretical models of nerve membranes. Biophysical Journal, 1182:445-466, 1961.

[39] Y H Liu and X J Wang. Spike-frequency adaptation of a generalized leaky integrate-and-fire model neuron. Journal of Computational Neuroscience, pages 2545, 2001.

[40] C van Vreeswijk and D Hansel. Patterns of synchrony in neural networks with spike adaptation. Neural Computation, Jan 2001.

[41] A Compte, M V Sanchez-Vives, D A McCormick, and X-J Wang. Cellular and network mechanisms of slow oscillatory activity $(<1 \mathrm{~Hz})$ and wave propagation in a cortical network model. Journal of Neurophysiology, 89:2707-2725, 2003.

[42] A V Hill. Excitation and accommodation in nerve. Proceedings of the Royal Society of London. Series B, Biological Sciences, 119:305-355, 1936.

[43] S Coombes and M R Owen. Bumps, breathers, and waves in a neural network with spike frequency adaptation. Physical Review Letters, 94(148102), 2005.

[44] S Coombes and M R Owen. Exotic dynamics in a firing rate model of neural tissue with threshold accommodation. AMS Contemporary Mathematics, "Fluids and Waves: Recent Trends in Applied Analysis", 440:123$144,2007$.

[45] A van Rotterdam, F H Lopes da Silva, J van den Ende, M A Viergever, and A J Hermans. A model of the spatial-temporal characteristics of the alpha rhythm. Bulletin of Mathematical Biology, 44:283-305, 1982.

[46] F Grimbert. Mesoscopic models of cortical structures. PhD thesis, University of Nice-Sophia Antipolis, http://wwwsop.inria.fr/members/Francois.Grimbert/ docs/thesis_revised.pdf.zip, 2008.
[47] A Destexhe and T J Sejnowski. The Wilson-Cowan model, 36 years later. Biological Cybernetics, 101:1-2, 2009.

[48] G B Ermentrout and J D Cowan. A mathematical theory of visual hallucination patterns. Biological Cybernetics, 34:137-150, 1979.

[49] P C Bressloff, J D Cowan, M Golubitsky, P J Thomas, and $\mathrm{M}$ Wiener. Geometric visual hallucinations, Euclidean symmetry and the functional architecture of striate cortex. Philosophical Transactions of the Royal Society London B, 40:299-330, 2001.

[50] H R Wilson, R Blake, and S-H Lee. Dynamics of travelling waves in visual perception. Nature, 412:907-910, 2001.

[51] S-H Lee, R Blake, and D J Heeger. Travelling waves of activity in primary visual cortex during binocular rivalry. Nature Neuroscience, 8:22-23, 2005.

[52] S Wu, K Hamaguchi, and S Amari. Dynamics and computation of continuous attractors. Neural Computation, 20:994-1025, 2008.

[53] H G E Meijer, J Trojan, D Kleinböhl, R Hölz, and J R Buitenweg. A dynamic neural model of localization of brief successive stimuli in saltation. BMC Neuroscience, 10:P350, 2009.

[54] W Erlhagen and E Bicho. The dynamic neural field approach to cognitive robotics. Journal of Neural Engineering, 3:R36-R54, 2006.

[55] P C Bressloff. Les Houches Lectures in Neurophysics, chapter Pattern formation in visual cortex. SpringerVerlag, 2004.

[56] S Coombes, N A Venkov, L Shiau, I Bojak, D T J Liley, and C R Laing. Modeling electrocortical activity through improved local approximations of integral neural field equations. Physical Review E, 76:051901, 2007.

[57] P 1 Nunez. Neocortical Dynamics and Human EEG Rhythms. Oxford University Press, 1995.

[58] N A Venkov, S Coombes, and P C Matthews. Dynamic instabilities in scalar neural field equations with space-dependent delays. Physica D, 232:1-15, 2007.

[59] N A Venkov. Dynamics of Neural Field Models. PhD thesis, School of Mathematical Sciences, University of Nottingham, http:/ / www.umnaglava.org/pdfs.html, 2009. 
[60] P Grindrod and D Pinotsis. On the spectra of certain integro-differential-delay problems with applications in neurodynamics. Physica D, submitted, 2009.

[61] S Coombes, G J Lord, and M R Owen. Waves and bumps in neuronal networks with axo-dendritic synaptic interactions. Physica D, 178:219-241, 2003.

[62] K A Richardson, S J Schiff, and B J Gluckman. Control of traveling waves in the mammalian cortex. Physical Review Letters, 94:028103, 2005.

[63] J-Y Wu, X Huang, and C Zhang. Propagating Waves of Activity in the Neocortex: What They Are, What They Do. The Neuroscientist, 14(5):487-502, 2008.

[64] D J Pinto and G B Ermentrout. Spatially structured activity in synaptically coupled neuronal networks: I. Travelling fronts and pulses. SIAM Journal on Applied Mathematics, 62:206-225, 2001.

[65] C Laing and S Coombes. The importance of different timings of excitatory and inhibitory pathways in neural field models. Network: Computation in Neural Systems, 17:151 - 172, 2006.

[66] M L Steyn-Ross, D A Steyn-Ross, J W Sleigh, and D T J Liley. Theoretical electroencephalogram stationary spectrum for a white-noise-driven cortex: Evidence for a general anesthetic-induced phase transition. Physical Review E, 60:7299-7311, 1999.

[67] P A Robinson, C J Rennie, J J Wright, H Bahramali, E Gordon, and D 1 Rowe. Prediction of electroencephalographic spectra from neurophysiology. Physical Review E, 63:021903, 2001.

[68] I Bojak and D T J Liley. Modeling the effects of anesthesia on the electroencephalogram. Physical Review E, 71:041902, 2005.

[69] O Faugeras, F Grimbert, and J-J Slotine. Absolute stability and complete synchronization in a class of neural fields models. SIAM Journal on Applied Mathematics, 69:205-250, 2008.

[70] P C Bressloff and J D Cowan. The visual cortex as a crystal. Physica D, 173:226-258, 2002.

[71] P A Robinson. Patchy propagator, brain dynamics, and the generation of spatially structured gamma oscillations. Physical Review E, 73:041904, 2006.

[72] P C Bressloff. Traveling fronts and wave propagation failure in an inhomogeneous neural network. Physica D, 155, 2001.
[73] S Coombes and C R Laing. Neural fields with periodic modulation. Physical Review E, in preparation, 2009.

[74] V K Jirsa and J A S Kelso. Spatiotemporal pattern formation in neural systems with heterogeneous connection topologies. Physical Review E, 62:8462-8465, 2000.

[75] P C Bressloff. Spatially periodic modulation of cortical patterns by long-range horizontal connections. Physica D, 185:131-157, 2003.

[76] H Schmidt, A Hutt, and L Schimansky-Geier. Wave fronts in inhomogeneous neural field models. Physica D, 238:1101-1112, 2009.

[77] C A Brackley and M S Turner. Two-point heterogeneous connections in a continuum neural field model. Biological Cybernetics, 100:371-383, 2009.

[78] R Kötter and E Wanke. Mapping brains without coordinates. Philosophical Transactions of the Royal Society B, 360:751-766, 2005.

[79] K J Friston, L Harrison, and W Penny. Dynamic causal modelling. NeuroImage, 19:1273-1302, 2003.

[80] M Breakspear and V K Jirsa. Neuronal dynamics and brain connectivity. In V K Jirsa and A R McIntosh, editors, Handbook of Brain Connectivity, pages 3-64. Springer, 2007.

[81] P A Valdes-Sosa, J M Sanchez-Bornot, R C Sotero, Y Iturria-Medina, Y Aleman-Gomez Y, J BoschBayard, F Carbonell, and T Ozaki. Model driven EEG/fMRI fusion of brain oscillations. Human Brain Mapping, 30:2701-2721, 2009.

[82] V K Viktor Jirsa, K J Jantzen, A Fuchs, and J A Scott Kelso. Information Processing in Medical Imaging, chapter Neural Field Dynamics on the Folded ThreeDimensional Cortical Sheet and Its Forward EEG and MEG, pages 286-299. Springer Berlin, 2001.

[83] C J Honey, R Kötter, M Breakspear, and O Sporns. Network structure of cerebral cortex shapes functional connectivity on multiple time scales. Proceedings of the National Acadamy of Sciences USA National Academy of Sciences, USA, 104:10240-10245, 2007.

[84] A Ghosh, Y Rho, A R McIntosh, R Kötter, and V K Jirsa. Noise during rest enables the exploration of the brain's dynamic repertoire. PLoS Computational Biology, 4(10):e1000196, 2008.

[85] G Deco, V K Jirsa, A R McIntosh, O Sporns, and R Kötter. Key role of coupling, delay, and noise in resting brain fluctuations. Proceedings of the National Academy of Sciences, 106:10302-10307, 2009. 
[86] S Coombes. Dynamics of synaptically coupled integrate-and-fire-or-burst neurons. Physical Review E, 67:041910, 2003.

[87] Z P Kilpatrick and P C Bressloff. Effects of synaptic depression and adaptation on spatiotemporal dynamics of an excitatory neuronal network. Physica D, to appear, 2009.

[88] M Zachariou, D W N Dissanayake, S Coombes, M R Owen, and R Mason. Sensory gating and its modulation by cannabinoids: electrophysiological, computational and mathematical analysis. Cognitive Neurodynamics, pages 159-170, 2008.

[89] D T J Liley, P J Cadusch, M Gray, and P J Nathan. Drug induced modification of the system properties associated with spontaneous electroencephalographic activity. Physical Review E, 68:051096, 2003.

[90] B L Foster, I Bojak, and D T J Liley. Population based models of cortical drug response: insights from anaesthesia. Cognitive Neurodynamics, 2:283-296, 2008.

[91] P C Bressloff and J D Cowan. Spherical model of orientation and spatial frequency tuning in a cortical hypercolumn. Philosophical Transactions of the Royal Society $B, 358: 1643-1667,2003$.

[92] P Chossat and O Faugeras. Hyperbolic planforms in relation to visual edges and textures perception. http://arxiv.org/abs/0907.0963v2, 2009.

[93] O Faugeras, J Toubal, and B Cessac. A constructive mean-field analysis of multi population neural networks with random synaptic weights and stochastic inputs. Frontiers in Computational Neuroscience, 3, 2009.

[94] S Coombes. Neuronal networks with gap junctions: A study of piece-wise linear planar neuron models. SIAM Journal on Applied Dynamical Systems, 7:1101$1129,2008$.

[95] M Breakspear and S Knock. Kinetic models of brain activity. Brain Imaging and Behavior, 2:270-288, 2008.

[96] G Deco, V K Jirsa, P A Robinson, M Breakspear, and K J Friston. The dynamic brain: From spiking neurons to neural masses and cortical fields. PLoS Compututational Biology, 4:e1000092, 2008.

[97] D Q Nykamp and D Tranchina. A population density approach that facilitates large-scale modeling of neural networks: Extension to slow inhibitory synapses. Neural Computation, 13:511-546, 2001.
[98] D Cai, L Tao, M Shelley, and D W McLaughlin. An effective kinetic representation of fluctuation-driven neuronal networks with application to simple and complex cells in visual cortex. Proceedings of the $\mathrm{Na}$ tional Academy of Sciences of the United States of America, 101:7757-7762, 2004.

[99] F Apfaltrer, C Ly, and D Tranchina. Population density methods for stochastic neurons with realistic synaptic kinetics: Firing rate dynamics and fast computational methods. Network: Computation in Neural Systems, 17:373 - 418, 2006.

[100] I Ginzburg and H Sompolinsky. Theory of correlations in stochastic neural networks. Physical Review E, 50:3171-3191, 1994

[101] H Soula and C C Chow. Stochastic dynamics of a finite-size spiking neural network. Neural Computation, 19:3262-3292, 2007.

[102] S E Boustani and A Destexhe. A master equation formalism for macroscopic modeling of asynchronous irregular activity states. Neural Computation, 21:46-100, 2009.

[103] B Kriener, T Tetzlaff, A Aertsen, M Diesmann, and $S$ Rotter. Correlations and population dynamics in cortical networks. Neural Computation, 20:2185-2226, 2008. PMID: 18439141.

[104] M A Buice, J D Cowan, and C C Chow. Systematic fluctuation expansion for neural network activity equations. Neural Computation, to appear, 2009.

[105] P C Bressloff. Stochastic neural field theory and the system-size expansion. SIAM Journal on Applied Mathematics, submitted, 2009.

[106] E M Izhikevich and G M Edelman. Large-scale model of mammalian thalamocortical systems. Proceedings of the National Academy of Sciences, 105:3593-3598, 2008.

[107] S Coombes and M Zachariou. Coherent Behavior in Neuronal Networks, chapter Gap junctions and emergent rhythms. Springer, 2009. 